\title{
Carrier Frequency Offset Estimation Approach for Multicarrier Transmission on Hexagonal Time-Frequency Lattice
}

\author{
Kui Xu, Wenfeng Ma, Lianguo Wu, Wei Xie, Dongmei Zhang, Youyun Xu \\ Institute of Communications Engineering, PLAUST \\ E-mail:Igdxxukui@126.com
}

\begin{abstract}
In this paper, a novel carrier frequency offset estimation approach, including preamble structure, carrier frequency offset estimation algorithm, is proposed for hexagonal multi-carrier transmission (HMCT) system. The closed-form Cramer-Rao lower bound of the proposed carrier frequency offset estimation scheme is given. Theoretical analyses and simulation results show that the proposed preamble structure and carrier frequency offset estimation algorithm for HMCT system obtains an approximation to the Cramer-Rao lower bound mean square error (MSE) performance over the doubly dispersive (DD) propagation channel.
\end{abstract}

Index Terms-Hexagonal Multi-Carrier Transmission System; Preamble Structure; Carrier Frequency Offset Estimation; Cramer-Rao Lower Bound;

\section{INTRODUCTION}

Orthogonal frequency division multiplexing (OFDM) systems with guard-time interval or cyclic prefix can prevent inter-symbol interference (ISI). OFDM has overlapping spectra and rectangular impulse responses. Consequently, each OFDM sub-channel exhibits a sinc-shape frequency response. Therefore, the time variations of the channel during one OFDM symbol duration destroy the orthogonality of different subcarriers, and result in power leakage among subcarriers, known as intercarrier interference (ICI), which causes degradation in system performance. In order to overcome the above drawbacks of OFDM system, several pulse-shaping OFDM systems were proposed [1]-[8].

It is shown that signal transmission through a rectangular lattice is suboptimal for doubly dispersive (DD) channel [9][13]. By using results from sphere covering theory, the authors have demonstrated that hexagonal multi-carrier transmission (HMCT) system obtains lower energy perturbation than OFDM system, hence providing better performance against ISI/ICI [9]-[11]. There are mainly two types of HMCT systems, (1): HMCT system with orthogonal prototype pulse [8]-[10], named as lattice OFDM (LOFDM) system, which confines the transmission pulses to a set of orthogonal ones. (2): HMCT system with nonorthogonal prototype pulse [11][13], named as hexagonal multicarrier transmission (HMT) system, which abandons the orthogonality condition of the modulated pulses and obtains the high robustness performance on combating the dispersion of DD propagation channel in both time and frequency domain.
To optimally combat the impact of the DD propagation channels, the lattice parameters in the time-frequency ( $\mathrm{T}$ F) plane and the pulse shape of modulation waveform of HMCT system are jointly optimized to adapt to the channel scattering function. It is shown in [14]-[17] that the HMCT system outperforms OFDM system from the robustness against channel dispersion point of view.

The basic mathematical operation of the received signal performed by the demodulator is a projection onto an identically structured function set generated by the prototype pulse function [5], i.e. an optimal match filter. In HMCT system, there is no cyclic prefix and data symbols of HMCT signal are transmitted on hexagonal lattice points in the T-F plane. Traditional timing and frequency synchronization schemes can not be applied to HMCT system directly. In our previous work [14]-[17], signal to interference and noise ratio (SINR) of HMCT system over DD channel is analyzed and the MaxSINR based timing synchronization scheme is proposed.

In this paper, a novel preamble structure and two carrier frequency offset estimation algorithms, named as phase differential algorithm and least squares algorithm, for HMCT system are proposed. After detailed derivation, the close form performance lower bound of the proposed carrier frequency offset estimation algorithm is given. Theoretical analyses and simulation results show that the proposed carrier frequency estimation approach for HMCT system obtains an approximation to the lower bound mean square error (MSE) performance over DD channel.

\section{Hexagonal Multi-CARrier Transmission SYSTEM}

In HMCT systems, the transmitted baseband signal can be expressed as [11]-[13]

$x(t)=\sum_{m} \sum_{n=0}^{\frac{N}{2}-1} c_{m, 2 n} \psi_{m, 2 n}(t)+\sum_{m} \sum_{n=0}^{\frac{N}{2}-1} c_{m, 2 n+1} \psi_{m, 2 n+1}(t)$

where $T$ and $F$ are the lattice parameters, which can be viewed as the symbol period and the subcarrier separation, respectively; $c_{m, n}$ denotes the transmitted data symbol, which is assumed to be taken from a specific signal constellation and independent and identically distributed (i.i.d.) with zero 
mean and average power $\sigma_{c}^{2} ; m \in \mathcal{M}$ and $n \in \mathcal{N}$ are the position indices in the T-F plane; $\mathcal{M}$ and $\mathcal{N}$ denote the sets from which $m, n$ can be taken, with cardinalities $M$ and $N$, respectively. $\psi_{m, 2 n}(t)=\psi(t-m T) e^{j 2 \pi n F t}$ and $\psi_{m, 2 n+1}(t)=\psi\left(t-m T-\frac{T}{2}\right) e^{j 2 \pi\left(n F+\frac{F}{2}\right) t}$ are the transmitted pulses generated by the prototype pulse $\psi(t)$. The ambiguity function of the prototype pulse is defined as

$$
A_{\psi}(\tau, v)=\int_{-\infty}^{\infty} \psi(t) \psi^{*}(t-\tau) e^{-j 2 \pi v t} d t
$$

The baseband DD channel can be modeled as a random linear operator $\mathrm{H}$ [18]

$$
\mathrm{H}[x(t)]=\int_{0}^{\tau_{\max }} \int_{-f_{d}}^{f_{d}} H(\tau, v) x(t-\tau) e^{j 2 \pi v t} d \tau d v
$$

where $\tau_{\max }$ and $f_{d}$ are the maximum multipath delay spread and the maximum Doppler frequency, respectively [19]. $H(\tau, v)$ is called the delay-Doppler spread function, which is the Fourier transform of the time-varying impulse response of the channel $h(t, \tau)$ with respect to $t$ [18].

In wide-sense stationary uncorrelated scattering (WSSUS) assumption, the DD channel is characterized by the secondorder statistics

$$
\mathrm{E}\left[H(\tau, v) H^{*}\left(\tau_{1}, v_{1}\right)\right]=S_{H}(\tau, v) \delta\left(\tau-\tau_{1}\right) \delta\left(v-v_{1}\right)
$$

where E[·] denotes the expectation and $S_{H}(\tau, v)$ is called the scattering function, which characterizes the statistics of the WSSUS channel. Without loss of generality, $H(\tau, v)$ is assumed to have zero mean and unit variance, i.e., $\int_{0}^{\tau_{\max }} \int_{-f_{d}}^{f_{d}} S_{H}(\tau, v) d \tau d v=1$. The received baseband signal can be expressed as

$$
r(t)=\mathrm{H}[x(t)]+w(t)
$$

where $w(t)$ is the AWGN with variance $\sigma_{w}^{2}$.

The basic mathematical operation of the received signal performed by the demodulator is a projection onto an identically structured function set generated by the prototype pulse function, i.e. an optimal match filter [17]. To recover the transmitted data symbol $\hat{c}_{m, 2 n}$, the match filter receiver projects the received signal $r(t)$ on prototype pulse function $\psi_{m, 2 n}(t)$, i.e., $\hat{c}_{m, 2 n}=\left\langle r(t), \psi_{m, 2 n}(t)\right\rangle=\int_{-\infty}^{\infty} r(t) \psi_{m, 2 n}^{*}(t) d t$, where $\langle\cdot\rangle$ denotes the inner product and $(\cdot)^{*}$ denotes the complex conjugate. Hence, frequency synchronization plays a critical role in ensuring reliable demodulation. Firstly, we will analyze the effects of carrier frequency offset on the recovered data symbol $\hat{c}_{m, 2 n}$.

\section{EFFects of CARRIER FREQUency OfFSET ON HMCT SYSTEM}

Under the assumption that there is a carrier frequency offset $\Delta f$ between the received signal $r(t)$ and the transmitted signal $x(t)$. After ignoring the impact of additive noise, the received signal $r(t)$ can be written as

$$
r(t)=e^{j 2 \pi \Delta f t} \int_{0}^{\tau_{\max }} \int_{-f_{d}}^{f_{d}} H(\tau, v) x(t-\tau) e^{j 2 \pi v t} d \tau d v
$$

and the recovered data symbol $\hat{c}_{m, 2 n}$ can be expressed as

$$
\begin{aligned}
\hat{c}_{m, 2 n} & =\left\langle r(t), \psi_{m, 2 n}(t)\right\rangle \\
& =\sum_{m^{\prime}} \sum_{n^{\prime}=0}^{N / 2-1} c_{m^{\prime}, 2 n^{\prime}} \Xi_{m, n ; m^{\prime}, 2 n^{\prime}}^{\Delta f} \\
& +\sum_{m^{\prime}} \sum_{n^{\prime}=0}^{N / 2-1} c_{m^{\prime}, 2 n^{\prime}+1} \Xi_{m, n ; m^{\prime}, 2 n^{\prime}+1}^{\Delta f}
\end{aligned}
$$

where $\Xi_{m, n ; m^{\prime}, 2 n^{\prime}}^{\Delta f}$ in (7) can be expressed as (8) at the top of the next page.

Let $m=m^{\prime}$ and $n=n^{\prime}, \Xi_{m, 2 n ; m, 2 n}^{\Delta f}$ can be expressed

$$
\begin{aligned}
\Xi_{m, 2 n ; m, 2 n}^{\Delta f} & =e^{-j 2 \pi m T \Delta f} \int_{0}^{\tau_{\max }} \int_{-f_{d}}^{f_{d}} A_{\psi}^{*}(\tau, v+\Delta f) \\
& \cdot H(\tau, v) e^{-j 2 \pi n F \tau} e^{j 2 \pi v m T} d \tau d v \\
& =e^{-j 2 \pi m T \Delta f} A_{H}\left(\tau_{\max }, f_{d}, \Delta f\right)
\end{aligned}
$$

and $\Xi_{m, n ; m^{\prime}, 2 n^{\prime}+1}^{\Delta f}$ in (7) can be expressed as (10) at the top of the next page.

Let $m=m^{\prime}$ and $n=n^{\prime}, \Xi_{m, 2 n ; m, 2 n+1}^{\Delta f}$ can be expressed as

$$
\begin{aligned}
\Xi_{m, 2 n ; m, 2 n+1}^{\Delta f} & =e^{j \pi m F T} e^{-j 2 \pi m T \Delta f} \int_{0}^{\tau_{\max }} \int_{-f_{d}}^{f_{d}} \\
& \cdot A_{\psi}^{*}(\tau, v+\Delta f) H(\tau, v) e^{-j 2 \pi\left(n+\frac{1}{2}\right) F \tau} \\
& \cdot e^{j 2 \pi v\left(m+\frac{1}{2}\right) T} d \tau d v
\end{aligned}
$$

Hence, the data symbol $\hat{c}_{m, 2 n}$ in (7) can be rewritten as

$$
\begin{aligned}
\hat{c}_{m, 2 n} & =c_{m, 2 n} \Xi_{m, 2 n ; m, 2 n}^{\Delta f}+\sum_{n^{\prime}=0, n^{\prime} \neq n}^{N / 2-1} c_{m, 2 n^{\prime}} \Xi_{m, 2 n ; m, 2 n^{\prime}}^{\Delta f} \\
& +\sum_{m^{\prime} \neq 0} \sum_{n^{\prime}=0}^{N / 2-1} c_{m^{\prime}, 2 n^{\prime}} \Xi_{m, 2 n ; m^{\prime}, 2 n^{\prime}}^{\Delta f} \\
& +\sum_{m^{\prime}} \sum_{n^{\prime}=0}^{N / 2-1} c_{m^{\prime}, 2 n^{\prime}+1} \Xi_{m, 2 n ; m^{\prime}, 2 n^{\prime}+1}^{\Delta f}
\end{aligned}
$$

The first term in equation (12) represents the desired symbol and the last three terms denote ISI/ICI. Concerning the useful portion, the transmitted symbols $c_{m, 2 n}$ are attenuated by $A_{H}\left(\tau_{\max }, f_{d}, \Delta f\right)$ which is caused by the carrier frequency offset $\Delta f$ and doubly dispersive channel. Meanwhile, the transmitted symbols rotated by a phasor $-j 2 \pi m T \Delta f$.

\section{The Proposed Preamble Structure}

The proposed preamble is composed of two training sequences $\boldsymbol{P}_{1}$ and $\boldsymbol{P}_{2}$ in the frequency domain, as depicted in Fig. 1. $\boldsymbol{P}_{i}=\left[P_{i}(0), P_{i}(1), \cdots, P_{i}\left(N_{P}-1\right)\right], i \in\{1,2\}$ and $N_{P} \leq N / 2$ denotes the length of training sequence.

In this paper, $\boldsymbol{P}_{i}$ are selected as PN sequence and $\boldsymbol{P}_{1}=$ $\boldsymbol{P}_{2}$. Assume that the index of the training sequence is $l \in$ 


$$
\begin{aligned}
\Xi_{m, 2 n ; m^{\prime}, 2 n^{\prime}}^{\Delta f} & =\int_{-\infty}^{\infty} \int_{0}^{\tau_{\max }} \int_{-f_{d}}^{f_{d}} e^{j 2 \pi \Delta f t} \psi_{m^{\prime}, 2 n^{\prime}}(t-\tau) H(\tau, v) \psi_{m, 2 n}^{*}(t) e^{j 2 \pi v t} d \tau d v d t \\
& =e^{-j 2 \pi F T m\left(n-n^{\prime}\right)} e^{-j 2 \pi m T \Delta f} \int_{0}^{\tau_{\max }} \int_{-f_{d}}^{f_{d}} A_{\psi}^{*}\left(\left(m^{\prime}-m\right) T+\tau,\left(n^{\prime}-n\right) F+v+\Delta f\right) \\
& \cdot H(\tau, v) e^{-j 2 \pi n^{\prime} F \tau} e^{j 2 \pi v m T} d \tau d v
\end{aligned}
$$

$$
\begin{aligned}
\Xi_{m, 2 n ; m^{\prime}, 2 n^{\prime}+1}^{\Delta f} & =\int_{-\infty}^{\infty} \int_{0}^{\tau_{\max }} \int_{-f_{d}}^{f_{d}} e^{j 2 \pi \Delta f t} \psi_{m^{\prime}, 2 n^{\prime}+1}(t-\tau) H(\tau, v) \psi_{m, 2 n}^{*}(t) e^{j 2 \pi v t} d \tau d v d t \\
= & e^{-j 2 \pi F T m\left(n-n^{\prime}\right)} e^{-j 2 \pi m T \Delta f} \int_{0}^{\tau_{\max }} \int_{-f_{d}}^{f_{d}} A_{\psi}^{*}\left(\left(m^{\prime}-m\right) T+\tau,\left(n^{\prime}+\frac{1}{2}-n\right) F+v+\Delta f\right) \\
& \cdot H(\tau, v) e^{-j 2 \pi\left(n^{\prime}+\frac{1}{2}\right) F \tau} e^{j 2 \pi v m T} d \tau d v
\end{aligned}
$$



Tra. Seq. 1 Tra. Seq. 2 Payload

Fig. 1. The proposed preamble structure.

$\left[0,2 N_{P}-1\right]$, the frequency domain training sequence can be expressed as

$$
D_{1, l}= \begin{cases}P_{1}(\lfloor l / 2\rfloor), & l \in\left[0,2 N_{P}-1\right] \\ 0 & , \text { else }\end{cases}
$$

where $\lfloor\cdot\rfloor$ denotes the floor function.

\section{The Proposed Carrier Frequency Offset ESTIMATION ALGORITHM}

We assume that the received signal $r(t)$ experiences a carrier frequency offset $\Delta f$ and the timing offset is completely compensated. The demodulated training sequences can be expressed as

$$
\begin{aligned}
\hat{D}_{1,2 l} & =D_{1,2 l} \Xi_{1,2 l ; 1,2 l}^{\Delta f}+\sum_{l^{\prime}=0, l^{\prime} \neq l}^{N / 2-1} D_{1,2 l^{\prime}} \Xi_{1,2 l ; 1,2 l^{\prime}}^{\Delta f} \\
& +\sum_{k^{\prime} \neq 1} \sum_{l^{\prime}=0}^{N / 2-1} D_{k^{\prime}, 2 l^{\prime}} \Xi_{k, 2 l ; k^{\prime}, 2 l^{\prime}}^{\Delta f} \\
& +\sum_{k^{\prime}} \sum_{l^{\prime}=0}^{N / 2-1} D_{k^{\prime}, 2 l^{\prime}+1} \Xi_{k, 2 l ; k^{\prime}, 2 l^{\prime}+1}^{\Delta f}+W_{0,2 l}
\end{aligned}
$$

and

$$
\begin{aligned}
\hat{D}_{1,2 l+1} & =D_{1,2 l+1} \Xi_{1,2 l+1 ; 1,2 l+1}^{\Delta f} \\
& +\sum_{l^{\prime}=0, l^{\prime} \neq l}^{N / 2-1} D_{1,2 l^{\prime}+1} \Xi_{1,2 l+1 ; 1,2 l^{\prime}+1}^{\Delta f} \\
& +\sum_{k^{\prime} \neq 1} \sum_{l^{\prime}=0}^{N / 2-1} D_{k^{\prime}, 2 l^{\prime}+1} \Xi_{k, 2 l+1 ; k^{\prime}, 2 l^{\prime}+1}^{\Delta f} \\
& +\sum_{k^{\prime}} \sum_{l^{\prime}=0}^{N / 2-1} D_{k^{\prime}, 2 l^{\prime}} \Xi_{k, 2 l+1 ; k^{\prime}, 2 l^{\prime}}^{\Delta f}+W_{0,2 l+1}
\end{aligned}
$$

$W_{k, 2 l}$ and $W_{k, 2 l+1}$ denote the AWGN in frequency domain. Thanks to the central limit theorem [22], the last four terms in (14) and (15) can be denoted as $\phi_{1,2 l}$ and $\phi_{1,2 l+1}$, respectively. We can rewrite equation (14) and (15) as

$$
\begin{aligned}
\hat{D}_{1,2 l} & =D_{1,2 l} \Xi_{1,2 l ; 1,2 l}^{\Delta f}+\phi_{1,2 l} \\
& =D_{1,2 l} e^{-j 2 \pi T^{\prime} \Delta f} \iint h(\tau, v) A_{\psi}^{*}(\tau, v+\Delta f) \\
& \cdot e^{-j 2 \pi 2 l F^{\prime} \tau} e^{j 2 \pi v T^{\prime}} d \tau d v+\phi_{1,2 l}
\end{aligned}
$$

and

$$
\begin{aligned}
\hat{D}_{1,2 l+1} & =D_{1,2 l+1} \Xi_{1,2 l+1 ; 1,2 l+1}^{\Delta f}+\phi_{1,2 l+1} \\
& =D_{1,2 l+1} e^{-j 2 \pi\left(1+\frac{1}{2}\right) T^{\prime} \Delta f} \iint_{f} h(\tau, v) \\
& \cdot A_{\psi}^{*}(\tau, v+\Delta f) e^{-j 2 \pi(2 l+1) F^{\prime} \tau} \\
& \cdot e^{j 2 \pi v(1+1 / 2) T^{\prime}} d \tau d v+\phi_{1,2 l+1}
\end{aligned}
$$

We assume that $D_{1,2 l} D_{1,2 l}^{*}=\sigma_{s}^{2}$ and define $\gamma_{m}$ as (18) at the top of next page. $\vartheta_{0}=\pi T^{\prime} \Delta f$ denotes the phase rotation caused by frequency offset and $\vartheta_{1}$ is a constant. Let $\varepsilon=\Delta f T_{s} N / 2$ denotes the normalized frequency offset, and $T_{s}$ denotes the sample period. $W$ is the additive noise with zero mean and average power $\sigma_{W}^{2}$. There are two fine frequency offset estimation schemes: Phase differential estimation scheme and least squares estimation scheme. 


$$
\begin{aligned}
\gamma_{m} & =\sum_{l=0}^{N / 2-1} \hat{D}_{1,2 l+2 m+1} \exp \left(j \cdot \arg \left(D_{1,2 l+2 m+1}^{*}\right)\right)\left(\hat{D}_{1,2 l} \exp \left(j \cdot \arg \left(D_{1,2 l}^{*}\right)\right)\right)^{*} \\
& =\sum_{l=0}^{N / 2-1}\left\{\sigma_{s}^{2} e^{-j \pi T^{\prime} \Delta f} \sum_{i=0}^{L-1} e^{-j 2 \pi(2 m+1) i F^{\prime}} \int_{-f_{d}}^{f_{d}} S(i, v)\left|A_{\psi}^{*}(i, v+\Delta f)\right|^{2} e^{j \pi v T^{\prime}} d v+W_{m}\right\} \\
& \simeq e^{-j \vartheta_{0}} e^{-j(m+1) \vartheta_{1}} \sum_{l=0}^{N / 2-1}\left\{\left.\sigma_{s}^{2}\left|\sum_{i=0}^{L-1} e^{-j 2 \pi(2 m+1) i F^{\prime}} \int_{-f_{d}}^{f_{d}} S(i, v)\right| A_{\psi}^{*}(i, v+\Delta f)\right|^{2} e^{j \pi v T^{\prime}} d v \mid\right\}+W
\end{aligned}
$$

\section{A. Phase Differential Carrier Frequency Offset Estimation Algorithm}

The phase differential carrier frequency offset estimation algorithm can be expressed as

$$
\begin{aligned}
\hat{\varepsilon} & =\frac{N}{2 \pi M}\left(\arg \left(\gamma_{0}\right)\right. \\
& \left.-\frac{1}{N / 2-1} \sum_{m=1}^{N / 2-1}\left(\arg \left(\gamma_{m}\right)-\arg \left(\gamma_{m-1}\right)\right)\right)
\end{aligned}
$$

The phase differential estimation scheme is valid for $\varepsilon \in$ $[-N / 2 M, N / 2 M]=[-\rho / 2, \rho / 2]$, and $\rho$ denotes system spectral efficiency.

\section{B. Least Squares Carrier Frequency Offset Estimation Algo-} rithm

We can model the phase of $\gamma_{m}$ as

$$
\arg \left(\gamma_{m}\right) \simeq \vartheta_{0}+(m+1) \vartheta_{1}+\zeta_{m}
$$

and

$$
\arg (\vec{\gamma}) \simeq \mathbf{C} \vec{\vartheta}+\vec{\zeta}
$$

where $\vec{\gamma}=\left[\gamma_{0}, \gamma_{1}, \cdots, \gamma_{N / 2-1}\right]$ and $\arg (\vec{\gamma})=$ $\left[\arg \left(\gamma_{0}\right), \arg \left(\gamma_{1}\right), \cdots, \arg \left(\gamma_{N / 2-1}\right)\right]^{T}, \quad \vec{\vartheta}=\left[\vartheta_{0}, \vartheta_{1}\right]$ and $\vec{\zeta}=\left[\zeta_{0}, \zeta_{1}, \cdots, \zeta_{N / 2-1}\right] . \mathbf{C}$ in (21) can be expressed as

$$
\mathbf{C}=\left[\begin{array}{cc}
1 & 0 \\
1 & 1 \\
\vdots & \vdots \\
1 & N / 2-1
\end{array}\right]
$$

The least squares carrier frequency offset estimation algorithm can be expressed as

$$
\hat{\vec{\vartheta}}=\left(\mathbf{C}^{T} \mathbf{C}\right)^{-1} \mathbf{C}^{T} \arg (\vec{\gamma})
$$

and $\left(\mathbf{C}^{T} \mathbf{C}\right)^{-1}$ in (23) can be obtained by

$$
\left(\mathbf{C}^{T} \mathbf{C}\right)^{-1}=\frac{2}{N \Delta m^{2}}\left[\begin{array}{cc}
\overline{m^{2}} & -\bar{m} \\
-\bar{m} & 1
\end{array}\right]
$$

where $\bar{m}=2 / N \sum_{k=0}^{N / 2-1} k, \overline{m^{2}}=2 / N \sum_{k=0}^{N / 2-1} k^{2}$ and $\Delta m^{2}=2 / N \sum_{k=0}^{N / 2-1}(k-\bar{m})^{2}$. Hence, the estimation of $\hat{\vec{\vartheta}}$ can be rewritten as

$$
\hat{\vec{\vartheta}}=\left[\hat{\vartheta}_{0}, \hat{\vartheta}_{1}\right]^{T}=\frac{1}{\Delta m^{2}}\left[\begin{array}{cc}
\bar{\gamma} \overline{m^{2}} & \bar{m}(\overline{\gamma m}) \\
\overline{\gamma m} & \bar{m} \cdot \bar{\gamma}
\end{array}\right]
$$

where $\bar{\gamma}=2 / N \sum_{k=0}^{N / 2-1} \arg \left(\gamma_{k}\right)$ and $\overline{\gamma m}=$ $2 / N \sum_{k=0}^{N / 2-1} \arg \left(\gamma_{k} k\right)$. The phase rotation $\vartheta_{0}$ caused by frequency offset and the constant $\vartheta_{1}$ can be obtained by

$$
\hat{\vartheta}_{1}=\frac{\overline{\gamma m}-\bar{m} \cdot \bar{\gamma}}{\Delta m^{2}}
$$

and

$$
\hat{\vartheta}_{0}=\arg \left(\gamma_{0}\right)-\hat{\vartheta}_{1}=\frac{\bar{\gamma} m^{2}-\bar{m}(\overline{\gamma m})}{\Delta m^{2}}
$$

The normalized frequency offset $\hat{\varepsilon}$ can be expressed as

$$
\hat{\varepsilon}=\frac{N\left(\arg \left(\gamma_{0}\right)-\hat{\vartheta}_{1}\right)}{2 \pi M}
$$

The least squares frequency offset estimation scheme is valid for $\varepsilon \in[-N / 2 M, N / 2 M]=[-\rho / 2, \rho / 2]$.

\section{Simplified Carrier Frequency Offset Estimation Scheme}

Let $\boldsymbol{\Psi}_{0}=\left[\psi_{0}(0), \psi_{0}(1), \cdots, \psi_{0}\left(L_{\psi}-1\right)\right]$ denotes the prototype pulse with length $L_{\psi}$ in discrete HMCT system. In order to recover the transmitted frequency domain training sequence $D_{1, l}$, we need $N_{p}$ inner product operations with $L_{\psi}$ points, which introduce high computational complexity.

Theorem 1: Let $\boldsymbol{r}=\left[r(0), r(1), \cdots, r\left(L_{\psi}-1\right)\right]$ denote the received signal vector with length $L_{\psi}$, and $\boldsymbol{\Phi}=\left\{\boldsymbol{\Psi}_{n}\right\}, n=$ $0,1, \cdots, N / 2-1$, denotes the objective projection subspace. $\boldsymbol{\Psi}_{n}=\left[\psi_{n}(0), \psi_{n}(1), \cdots, \psi_{n}\left(L_{\psi}-1\right)\right]$ and satisfies

$$
\psi_{n}(m)=\psi_{0}(m) e^{\frac{j 2 \pi m n}{N / 2}}
$$

The time frequency subspace projection of the received signal $\boldsymbol{r}$ on the objective projection subspace $\boldsymbol{\Phi}$ is equivalent to $\operatorname{FFT}\left(\sum_{l=0}^{Q_{\psi}-1} r_{\psi}(k+l N / 2)\right), k=0,1, \cdots, N / 2-1 . Q_{\psi}=$ $\left\lceil\frac{L_{\psi}}{N / 2}\right\rceil$ and $\lceil\cdot\rceil$ denotes the ceiling function. $\boldsymbol{r}_{\psi}=\boldsymbol{r} \odot \boldsymbol{\Psi}_{0}^{*}$ and $\boldsymbol{r}_{\psi}=\left[r_{\psi}(0), r_{\psi}(1), \cdots, r_{\psi}\left(L_{\psi}-1\right)\right] . \odot$ denotes the Hadamard product and $\operatorname{FFT}(\cdot)$ denotes the Fast Fourier Transform.

Proof: The output symbol $Y(i)$ of the time frequency subspace projector after projecting the received signal $\boldsymbol{r}$ to the $i$ th subspace $\boldsymbol{\Psi}_{i}$ can be expressed as

$$
\begin{aligned}
Y(i) & =\left\langle\boldsymbol{r}, \boldsymbol{\Psi}_{i}\right\rangle=\sum_{n=0}^{L_{\psi}-1} r(n) \psi_{i}^{*}(n) \\
& =\sum_{n=0}^{L_{\psi}-1} r(n) \psi_{0}^{*}(n) e^{\frac{-j 2 \pi n i}{N / 2}}
\end{aligned}
$$


After performing the IFFT on the signal $Y(i)$, the transformed signal can be expressed as

$$
\begin{aligned}
y(k) & =\sum_{i=0}^{N / 2-1} Y(i) e^{\frac{j 2 \pi k i}{N / 2}} \\
& =\frac{1}{N / 2} \sum_{i=0}^{N / 2-1} \sum_{n=0}^{L_{\psi}-1} r(n) \psi_{0}^{*}(n) e^{\frac{j 2 \pi(k-n) i}{N / 2}} \\
& =\frac{1}{N / 2} \sum_{i=0}^{N / 2-1} \sum_{n=0}^{N / 2-1} \sum_{l=0}^{Q_{\psi}-1} r_{\psi}\left(n+\frac{l N}{2}\right) e^{\frac{j 2 \pi(k-n) i}{N / 2}} \\
& =\sum_{l=0}^{Q_{\psi}-1} r_{\psi}\left(k+\frac{l N}{2}\right)
\end{aligned}
$$

where $\lceil\cdot\rceil$ denotes the ceiling function. $r_{\psi}(n)=r(n) \psi_{0}^{*}(n)$, $n=0,1, \cdots, L_{\psi}-1$, which can be denoted as $\boldsymbol{r}_{\psi}=\boldsymbol{r} \odot \boldsymbol{\Psi}_{0}^{*}$.

We can conclude from Theorem 1 that the transmitted frequency domain training sequence $D_{1, l}$ can be obtained by the following two steps. The first step is to calculate the Hadamard product of the received signal $\boldsymbol{r}$ and the prototype pulse $\boldsymbol{\Psi}_{0}$, that is $\boldsymbol{r}_{\psi}$. The second step is to superimpose vector $\boldsymbol{r}_{\psi}$ with period $N / 2$ and perform $N / 2$-point FFT on the superimposed vector $\mathbf{y}=[y(0), y(1), \ldots, y(N / 2)]$. Hence, two Hadamard product operations, two superimpose operators with period $N / 2$ and two $N / 2$-point FFT are needed to recover the transmitted frequency domain training sequence $D_{1, l}$, which is a low complexity approach compared to the traditional $N_{p}$ projectors approach.

\section{Cramer-Rao Lower Bound}

We can conclude from (19) and (28) that the proposed phase differential and least squares carrier frequency offset estimators are functions of $\gamma_{0} \cdot \gamma_{0}$ in AWGN channel can be expressed as

$$
\begin{aligned}
\gamma_{0} & =\sum_{l=0}^{N / 2-1}\left\{\sigma_{s}^{2} e^{-j \pi T^{\prime} \Delta f}+\phi_{1,2 l+1} \sigma_{s} e^{j 2 \pi T^{\prime} \Delta f}\right. \\
& \left.+\phi_{1,2 l}^{*} \sigma_{s} e^{-j 2 \pi(1+1 / 2) T^{\prime} \Delta f}+\phi_{1,2 l+1} \phi_{1,2 l}^{*}\right\} \\
& =\frac{N}{2} \sigma_{s}^{2} e^{-j 2 \pi M \varepsilon / N}+W_{0}
\end{aligned}
$$

The conditional probability density function $P\left(\gamma_{0} ; \varepsilon\right)$ can be expressed as

$$
\begin{aligned}
P\left(\gamma_{0} ; \varepsilon\right) & =\frac{1}{\left(\pi \sigma_{W}^{2}\right)^{1 / 2}} \exp \left\{-\frac{1}{\sigma_{W_{0}}^{2}}\left(\gamma_{0}-\frac{N}{2} \sigma_{s}^{2} e^{\frac{-j 2 \pi M \varepsilon}{N}}\right)\right. \\
& \left.\cdot\left(\gamma_{0}-\frac{N}{2} \sigma_{s}^{2} e^{\frac{-j 2 \pi M \varepsilon}{N}}\right)^{*}\right\}
\end{aligned}
$$

Differentiating the $\log$ likelihood function $\ln P\left(\gamma_{0} ; \varepsilon\right)$ with respect to $\varepsilon$, we have

$$
\begin{aligned}
\frac{\partial \ln P\left(\gamma_{0} ; \varepsilon\right)}{\partial \varepsilon} & =-\frac{1}{\sigma_{W_{0}}^{2}}\left\{\left(\gamma_{0}-\frac{N}{2} \sigma_{s}^{2} e^{\frac{-j 2 \pi M \varepsilon}{N}}\right)\right. \\
& \cdot\left(-j \pi M \sigma_{s}^{2} e^{\frac{j 2 \pi M \varepsilon}{N}}\right)+\left(\gamma_{0}-\frac{N}{2} \sigma_{s}^{2} e^{\frac{-j 2 \pi M \varepsilon}{N}}\right)^{*} \\
& \left.\cdot\left(j \pi M \sigma_{s}^{2} e^{\frac{-j 2 \pi M \varepsilon}{N}}\right)\right\}
\end{aligned}
$$

and

$$
\begin{aligned}
\frac{\partial^{2} \ln P\left(\gamma_{0} ; \varepsilon\right)}{\partial^{2} \varepsilon} & =-\frac{1}{\sigma_{W_{0}}^{2}}\left(\frac{2 \pi^{2} M^{2} \sigma_{s}^{2}}{N} \gamma_{0} e^{\frac{j 2 \pi M \varepsilon}{N}}\right. \\
& \left.+\frac{2 \pi^{2} M^{2} \sigma_{s}^{2}}{N} \gamma_{0}^{*} e^{\frac{-j 2 \pi M \varepsilon}{N}}\right)
\end{aligned}
$$

Hence, the Cramer-Rao lower bound of the proposed carrier frequency offset can be expressed as

$$
\begin{aligned}
\mathrm{E}\left\{|\hat{\varepsilon}-\varepsilon|^{2}\right\} & \geq-E\left[\frac{\partial^{2} \ln P\left(\gamma_{0} ; \varepsilon\right)}{\partial^{2} \varepsilon}\right]^{-1} \\
& =\frac{N}{2 \pi^{2} M^{2} \mathrm{SNR}}
\end{aligned}
$$

\section{Simulation Results}

In this section, we test the proposed synchronization approach for MCM system with hexagonal T-F lattice via computer simulations based on the discrete signal model. In the following simulations, the number of subcarriers for HMCT system is chosen as $N=40$, and the length of prototype pulse $L_{\psi}=600$. The center carrier frequency is $f_{c}=5 \mathrm{GHz}$ and the sampling interval is set to $T_{s}=10^{-6} \mathrm{~s}$. The system parameters of HMCT system are $F=25 \mathrm{kHz}, T=1 \times 10^{-4}$ s and signaling efficiency is set to $\rho=0.8$. WSSUS channel is chosen as DD channel with exponential power delay profile and U-shape Doppler spectrum.

The MSE performance of the proposed carrier frequency estimation algorithm over AWGN channel is given in Fig. 2. We can see from Fig. 2 that the proposed least squares carrier frequency estimation algorithm outperforms phase differential scheme at low SNR, and both the proposed schemes can obtain an approximation to the Cramer-Rao lower bound MSE performance.

The MSE performance of the proposed carrier frequency estimation algorithm over DD channel is given in Fig. 3. As shown in Fig. 3 that the proposed least squares carrier frequency estimation algorithm outperforms phase differential scheme at low SNR, but the phase differential scheme outperforms least squares algorithm at high SNR. The DD propagation channel introduces energy perturbation among the transmitted symbols, hence there is a gap between the MSE performance of the proposed two carrier frequency offset estimation algorithms and that of the Cramer-Rao lower bound. 


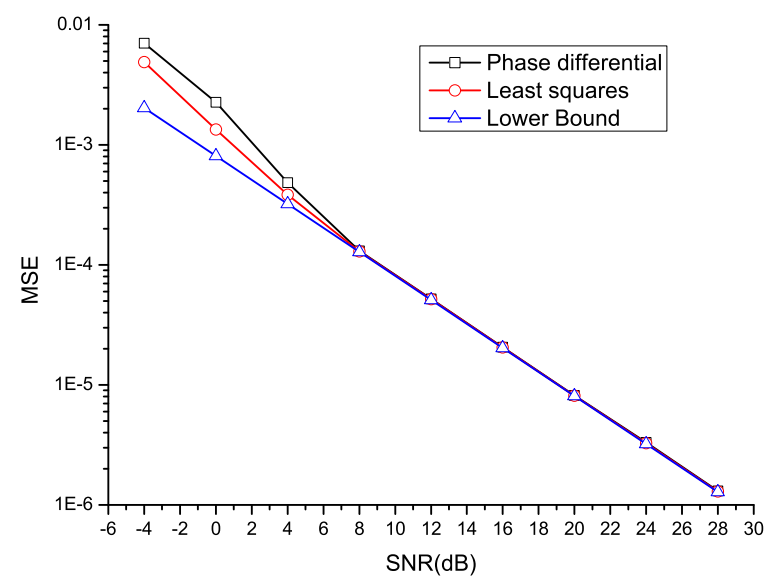

Fig. 2. MSE performance of the proposed carrier frequency estimation algorithm over AWGN channel.

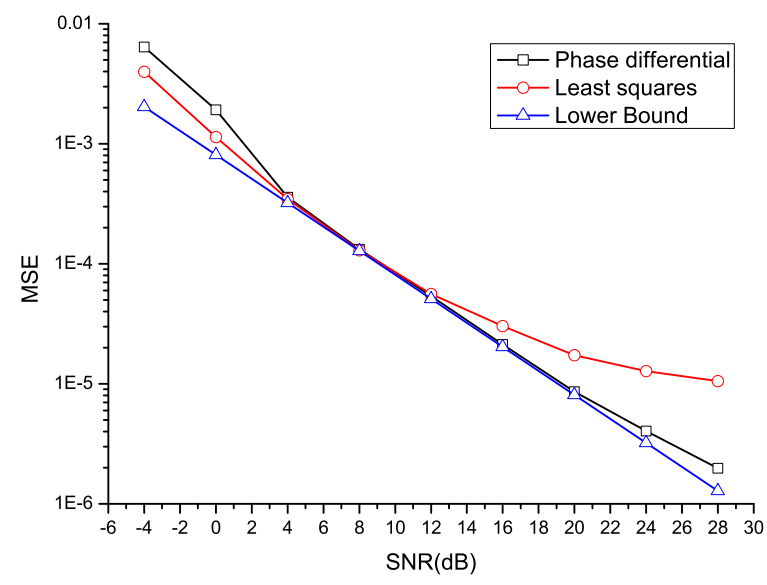

Fig. 3. MSE performance of the proposed carrier frequency estimation algorithm over DD channel.

\section{CONCLUSION}

In this paper, the effects of carrier frequency offset on HMCT system is analyzed. Meanwhile, a novel carrier frequency offset estimation approach, including preamble structure, carrier frequency offset estimation algorithm, is proposed for HMCT system. After detailed derivation, the close form performance lower bound of the proposed carrier frequency offset estimation alglrithm is given. Theoretical analyses and simulation results show that the proposed carrier frequency offset estimation approach for HMCT system obtains an approximation to the Cramer-Rao lower bound MSE performance over the DD channel with exponential power delay profile and U-shape Doppler spectrum.

\section{ACKNOWLEDGMENT}

This work was supported by the Jiangsu Province National Science Foundation for young scholars under Grant (No. BK2012055) and the Young Scientists Pre-research Fund of PLAUST under Grant (No. KYTYZLXY1211), the National Natural Science Foundation of China (No. 60972050) and the
Jiangsu Province National Science Foundation under Grant (No. BK2011002).

\section{REFERENCES}

[1] Kumbasar V., Kucur O. ICI reduction in OFDM systems by using improved sinc power pulse. Digital Signal Processing, vol.17, no.6, pp.997-1006, Nov. 2007.

[2] Das S., Schniter P. Max-SINR ISI/ICI-Shaping multicarrier communication over the doubly dispersive channel. IEEE Transactions on Signal Processing, vol.55, no.12, pp.5782-5795, Dec. 2007.

[3] Abbas H.K., Waleed A. M., Nihad S., The performance of multiwavelets based OFDM system under different channel conditions Digital Signal Processing, vol.20, no.2, pp.472-482, Mar. 2010.

[4] Gao X., Wang W., Xia X.G., et al. Cyclic prefixed OQAM-OFDM and its application to single-carrier FDMA. IEEE Transactions on Communications, vol.59, no.5, pp.1467-1480, May 2011.

[5] P. Jung, G. Wunder, The WSSUS pulse design problem in multicarrier transmission, IEEE Transactions on Communications, vol.55, no. 10, pp.1918-1928, Oct. 2007.

[6] G. Lin, L. Lundheim, N. Holte, Optimal pulses robust to carrier frequency offset for OFDM/QAM systems, IEEE Communications Letters, vol. 12, no. 3, pp. 161-163, Mar. 2008.

[7] P. Siohan, C. Siclet, N. Lacaille, Analysis and design of OFDM/OQAM systems based on filterbank theory, IEEE Transactions on Signal Processing, vol. 50, no. 5, pp. 1170-1183, May 2002.

[8] M. Ma, B. Jiao, C. Y. Lee, A dual-window technique for enhancing robustness of OFDM against frequency offset, IEEE Communications Letters, vol. 12, no. 1, pp. 17-19, Jan. 2008.

[9] Strohmer T, Beaver S. Optimal OFDM design for time-frequency dispersive channels. IEEE Trans. Commun. vol.51, no.7, pp.1111-1122, Jul. 2003.

[10] Yuan Z.G., Shen Y.H., A novel LOFDM signal and its optimization over doubly-dispersion channels. in Proc. 3rd IEEE Conference on Industrial Electronics and Applications, 2008. ICIEA 2008, pp.853-856, Jun. 2008.

[11] Han F.M., Zhang X.D. Hexagonal multicarrier modulation: A robust transmission scheme for time-frequency dispersive channels. IEEE Transactions on Signal Processing, vol.55, no.5, pp.1955-1961, May 2007.

[12] Han F.M., Zhang X.D., MLSD for hexagonal multicarrier transmission with time-frequency localized pulses, IEEE Transactions on Vehicular Technology, vol.58, no.3, pp.1598-1604, Mar. 2009.

[13] Han F.M., Zhang X.D., Asymptotic Equivalence of Two Multicarrier Transmission Schemes in Terms of Robustness Against TimeFrequency Dispersive Channels. IEEE Transactions on Vehicular Technology,vol.59, no.2, pp.1598-1604, Feb. 2010.

[14] Xu K., Shen Y. H., Effects of carrier frequency offset, timing offset, and channel spread factor on the performance of hexagonal multicarrier modulation systems. EURASIP Journal on Wireless Communications and Networking, vol.2009, pp.1-8, Jan. 2009.

[15] Xu K., Xu Y., Zhang D., SINR analysis of hexagonal multicarrier transmission systems in the presence of insufficient synchronization for doubly dispersive channel. Frequenz, vol.65, no.5, pp.149-157, Aug. 2011.

[16] Xu K., Lv Z., Xu Y., Zhang D., Max-SINR Based Timing Synchronization Scheme in Hexagonal Multicarrier Transmission. Wireless Personal Communications, DOI: 10.1007/s11277-012-0550-5, 2012.

[17] Xu K., Xu Y., Xia X., Zhang D., On Max-SINR Receiver for Hexagonal Multicarrier Transmission Over Doubly Dispersive Channel. to appear in Proc. IEEE GLOBECOM 2012.

[18] Bello P. A.,Characterization of randomly time-variant linear channels, IEEE Transactions on Communication System, vol.11, no.4, pp.360-393, Dec. 1963.

[19] L. Cohen, Time-frequency analysis. Englewood Cliffs, NJ: Prentice-Hall, 1995.

[20] P. Matthias, Mobile fading channels. West Sussex, England: John Wiley \& Sons, Ltd, 2002.

[21] J. H. Conway and N. J. A. Sloane, Sphere Packings, Lattices and Groups, 3rd ed. New York: Springer-Verlag, 1998.

[22] J. Proakis and M. Salehi, Digital Communications, 5th edition. New York: McGraw-Hill, 2007. 